\title{
Manuscript Consultants
}

The following consultants have provided valuable assistance in the reviewing of manuscripts during 1981:

Allen C. Alfrey Jose A.L. Arruda Tomas Berl Goeffrey Berlyne Thomas Berne Christopher R. Blagg Michael J. Blumenkrantz Wayne A. Border Nachman Brautbar John D. Brunzell Vito M. Campese Ralph Carmel Hugh Carroll Russell W. Chesney Jack Coburn Arthur Cohen John D. Conger Pirani Conrad Gbriel Danovitch Warren Davidson Ralph A. De Fronzo James Donadio Garabed Eknoyan Murray Epstein Eben I. Feinstein Richard N. Fine Stanley Franklin Eli A. Friedman Kenneth Gardner Richard J. Glassock John P. Hayslett Lee W. Henderson Richard Horton Willa Hsueh Michael Humphries Elaine Kaptein Michael Kirschenbaum Carl M.

Kjellstrand Charles R. Kleeman Oscar A. Kletsky James P. Knochel Joel D. Kopple Michael Koss Fredric Kraemer

Neal Kurtzman

John M. Leedom

Joseph Letteri

Nathan Levine

Francisco Llach

John F. Maher

Hartmut Malluche

Manuel Martinez-Maldonado

Franklin D. McDonald

Alfred F. Michael

Ronald Miller

Eddi Moore

John S. Najarian

Allen Nissenson

Karl D. Noph

Dimitrious G. Oreopoulous

Rufíno Pabico

A. Michael Parfitt

Robert A. Peraino

Victor E. Pollak

Warren R. Procci

Eberhard Ritz Sandra Sabatini Oscar Salvatierra Pat Schenfold Anthony Sebastian Alvin Sellers Fred Shapiro Frederick R. Singer Adrian Spitzer Richard L. Tannen Paul Tischan Carlos Vaamonde Darracot Vaughan Nick Vazeri Mackenzie Walser Ronald Wathen Michael Webber Robert Weiner Curtis B. Wilson Michael Ziegler Robert Zipser 